\title{
E3 ubiquitin ligase FBW7 $\alpha$ inhibits cholangiocarcinoma cell proliferation by downregulating c-Myc and cyclin $\mathbf{E}$
}

\author{
MING LI ${ }^{1-3}$, LING OUYANG ${ }^{2}$, ZHIGANG ZHENG $^{4}$, DAN XIANG $^{1}$, AIJUN TI $^{1}$, \\ LEIHUA LI ${ }^{1}$, YUZHEN DAN ${ }^{3}$, CHUNDONG YU $^{2,3}$ and WENGANG LI ${ }^{1,2}$ \\ ${ }^{1}$ Department of Hepatobiliary Pancreas and Vessel Surgery, Chenggong Hospital of Xiamen University;
${ }^{2}$ Xiamen City Key Laboratory of Biliary Tract Diseases, Chenggong Hospital of Xiamen University; ${ }^{3}$ State Key
Laboratory of Cellular Stress Biology, Innovation Center for Cell Signaling Network, School of Life Sciences,
Xiamen University, Xiamen; ${ }^{4}$ Department of General Surgery, the First Hospital of Fuzhou, Fuzhou, Fujian, P.R. China
}

Received August 19, 2016; Accepted January 23, 2017

DOI: $10.3892 /$ or.2017.5432

\begin{abstract}
FBW7 (F-box and WD repeat domain-containing 7), also known as CDC4, AGO and SEL10, is the substrate recognition component of an evolutionary conserved SCF (complex of SKP1, CUL1 and F-box protein)-type E3 ubiquitin ligase. It is a recognized tumor suppressor because it targets multiple oncoproteins for ubiquitination-mediated destruction and its mutations are frequently identified in a variety of human malignancies. However, the function of FBW7 in proliferation of cholangiocarcinoma (CCA) remains unknown. We found that overexpression of FBW7 $\alpha$ induced CCA cell arrest in G1 phase of cell cycle and inhibited cell proliferation in vitro and CCA xenograft tumor growth, suggesting that FBW7 $\alpha$ is a tumor suppressor in CCA progression. Overxpression of FBW7 $\alpha$ resulted in the protein degradation of its substrates such as c-Myc and cyclin $\mathrm{E}$ which promote CCA cell proliferation. Restoration of the expression of c-Myc, but not cyclin E, rescued the proliferation of FBW7 $\alpha$-overexpression CCA cells. These results suggest that FBW7 $\alpha$ plays an essential inhibitory role in CCA progression, indicating that targeting FBW7 $\alpha$ substrate c-Myc may be a potential strategy for CCA treatment.
\end{abstract}

\section{Introduction}

Cholangiocarcinoma (CCA) is one of the highly aggressive malignant tumors, arising from varying locations within

Correspondence to: Professor Chundong Yu, State Key Laboratory of Cellular Stress Biology, Innovation Center for Cell Signaling Network, School of Life Sciences, Xiamen University, Xiamen, Fujian 361005, P.R. China

E-mail: cdyu@xmu.edu.cn

Professor Wengang Li, Department of Hepatobiliary Pancreas and Vessel Surgery, Chenggong Hospital of Xiamen University, Xiamen, Fujian 361000, P.R. China

E-mail: lwg11861@163.com

Key words: F-box and WD repeat domain-containing 7, cholangiocarcinoma, proliferation, c-Myc, cyclin E ductular epithelium of biliary tree $(1,2)$. As the second most common primary hepatobiliary malignancy, CCA has very low postoperative 5-year survival rate, and treatment with radiotherapy and chemotherapy also carries poor overall survival rates $(1,2)$. Disappointingly, epidemiologic report showed that the overall incidence and mortality of CCA seem to be increasing (3). Hence, defining the molecular mechanisms of CCA is urgent and required for identifying early diagnosis and effective chemotherapy markers for CCA patients.

FBW7 (F-box and WD repeat domain-containing 7), also known as CDC4, AGO and SEL10, is the substrate recognition component of an evolutionary conserved SCF (complex of SKP1, CUL1 and F-box protein)-type E3 ubiquitin ligase $(4,5)$. Human FBW7 gene encodes three transcripts that are produced by alternative splicing and each mRNA consists of an isoform-specific first exon linked to ten shared exons, resulting in three protein isoforms (FBW7 $\alpha, \beta$ and $\gamma$ ) that only vary at the N-terminus (6). Three FBW7 isoforms have different subcellular location and tissue expression profile. FBW7 $\alpha, \beta$ and $\gamma$ locate in nucleoplasm, cytoplasm, and nucleolus, respectively (7). FBW7 $\alpha$ mRNA is expressed at much higher levels than either FBW7 $\beta$ or FBW7 $\gamma$ in most human tissues and varieties of exponentially growing human cells $(6,8)$. Consistently, adult mouse tissues express Fbw $7 \alpha$ mRNA ubiquitously, while the FBW7 $\beta$ displays higher levels in the brain and FBW7 $\gamma$ isoform exhibits an increased expression in muscle tissue (9). Grim et al utilized gene targeting to create isoform-specific Fbw7-null mutations in human colon cancer cell line HCT116 and found that FBW7 $\alpha$ was the major isoform that mediates the stability of cyclin E, c-Myc, and SREBP1 (sterol regulatory element binding protein 1) (8). These studies suggest that FBW7 $\alpha$ may play more important roles in most physiological and pathological process than other isoforms.

FBW7 targets multiple well-known oncoproteins including c-Myc (10), cyclin E (11), mTOR (12), Mcl-1 (13), Notch-1 (14,15) and AIB1 (16) for ubiquitination-mediated destruction, thus, it is a recognized tumor suppressor $(4,5)$. Consistent with the notion that FBW7 is a tumor suppressor in various human malignancies, FBW7 mutations are frequently identified in a variety of 
human malignancies. The frequency of FBW7 mutations in all primary human cancers analyzed was approximately $6 \%$ (87/1556). However, the mutation frequency in cholangiocarcinomas reached up to $35 \%$ (7/20) and the report showed that FBW7 hotspot mutants (4/7) in cholangiocarcinoma not only reveal defects in localization and substrate binding but also can abrogate wild-type FBW7 function through a dominant negative mechanism (17). It has been reported that the expression of FBW7 was downregulated in tumor tissues compared with adjacent non-tumorous tissues in intrahepatic (IHCC; 43 tumor specimens vs. 10 paired intrahepatic bile duct tissues) and perihilar (PHCC; 64 tumor specimens vs. 10 paired perihilar bile duct tissues) CCA (18). These results indicate that FBW7 is a general tumor suppressor in various cancers and it may play more important inhibitory roles in CCA progression. However, the function of FBW7 in the proliferation of CCA remains unknown. In the present study, we report that overexpression of FBW7 $\alpha$ inhibited CCA cell proliferatin in vitro and in vivo. Further study indicated that FBW7 $\alpha$ exerts antitumor activity in CCA at least in part through downregulating c-Myc and cyclin $\mathrm{E}$.

\section{Materials and methods}

Cell culture. CCA cell lines QBC-939 and MZ-cha1 were cultured in RPMI-1640 (HyClone) supplemented with $10 \%$ fetal bovine serum (Gibco) and $100 \mathrm{U} / \mathrm{ml}$ penicillin and $100 \mathrm{mg} / \mathrm{ml}$ streptomycin and were maintained in the humidified incubator with $95 \%$ air and $5 \% \mathrm{CO}_{2}$ at $37^{\circ} \mathrm{C}$. QBC-939 cells were obtained from Shuguang Wang (The Third Military Medical University, China); Mz-Cha1 cells were kindly provided by Dr Yabing Chen (University of Alabama at Birmingham, Birmingham, AL, USA).

Small interfering RNA and cell transfection. $\mathrm{p} 21^{\mathrm{CIP} / \mathrm{WAF} 1}$ siRNA (CUUCGACUUUGUCACCGAGdTdT) (19), FBW7 $\alpha$ siRNA-1 (GGGCAACA ACGACGCCGAAdTdT) (20), FBW $7 \alpha$ siRNA-2: (GUGAAGUUGUUGGAGUAGAdTdT) (21) and nonspecific siRNA were purchased from Invitrogen. siRNAs were transfected with X-tremeGENE transfection reagent to knock down $\mathrm{p} 21^{\mathrm{CIP} / \mathrm{WAF} 1}$ or FBW7 $\alpha$ expression in QBC-933 cells following the manufacturer's instructions.

Generation of lentiviruses and infection. The cDNA encoding FBW7 $\alpha$, c-Myc or cyclin E were cloned in the lentiviral vector pLVCS2.0. Oligonucleotide encoding shRNA targeting c-Myc or cyclin E or control oligonucleotide was cloned in the lentiviral vector GV112. The generation of lentivirus vectors was performed by co-transfecting pLV-CS2.0, GV112 or pLV-CS2.0/GV112 carrying the expression cassette with helper plasmids pVSV-G and pHR into $293 \mathrm{~T}$ cells. The viral supernatant was collected $48 \mathrm{~h}$ after transfection and CCA cells were infected with viral supernatants containing $10 \mu \mathrm{g} / \mathrm{ml}$ Polybrene for $24 \mathrm{~h}$ and then fresh medium was added to the infected cells. The shRNA target sequences are listed as follows: the target sequence for c-myc shRNA-1: CAAGGTA GTTATCCTTAAA; the target sequence for c-Myc shRNA-2: GTTGAAACACAAACTTGAA; the target sequence for cyclin E shRNA-1: ACATAGAGAACTGTGTCAA; the target sequence for cyclin E shRNA-2: AATTCTTCTGGATTGG
TTA; the target sequence for control shRNA: TTCTCCGA ACGTGTCACGT.

Western blot analysis. Briefly, Equal amounts of protein lysates were separated by SDS-PAGE and transferred onto PVDF membranes. After incubation with the primary antibody, the membranes were extensive washed by TBST buffer, then were incubated with horseradish peroxidase conjugated secondary antibody and visualized by chemiluminescence. Antibodies for FBW7 and c-Myc were purchased from Abcam; Antibodies for $\mathrm{p} 21^{\mathrm{CIP} / \mathrm{WAF} 1}$, mTOR, AIB1 and Notch1 were purchased from Cell Signaling Technology; Antibodies for cyclin E and Mcl-1 were purchased from Santa Cruz Biotechnology; Anti- $\beta$-actin antibody was purchased from Sigma.

Real-time RT-PCR. Briefly, total RNA was isolated with TRIzol reagent (Invitrogen) according to the manufacturer's instructions. Reverse transcription was performed using Revertra Ace qCR RT Master mix (Toyobo) with random primer and olig dT primer. Real-time PCR reactions were performed using FastStart Universal SYBR Green Master (Roche). Relative quantification was achieved by normalization to the amount of GAPDH. The primers used for real-time PCR are listed as follow: FBW7 $\alpha$ forward (22): GGAGATGG ACCAGGAGAGTG; FBW7 $\alpha$ reverse: GTTGGTGTTGC TGAACATGG; c-Myc forward: GCTGCTTAGACGCTG GATTT; c-Myc reverse: CACCGAGTCGTAGTCGAGGT; cyclin E forward: CTCCAGGAAGAGGAAGGCAA; cyclin E reverse: TCGATTTTGGCCATTTCTTCA; p21 $1^{\mathrm{CIP} / \mathrm{WAF} 1}$ forward: CAGGGGAGCAGGCTGAAG; $21^{\mathrm{CIP} / \mathrm{WAF} 1}$ reverse: GGATTAGGGCTTCCTCTTGG; GAPDH forward: CAC TCCTCCACCTTTGACGC; GAPDH reverse: TGCTGTAGC CAAATTCGTTGT.

CHX (cycloheximide) treatment. CCA cells were treated with $\mathrm{CHX}(60 \mu \mathrm{g} / \mathrm{ml})$ for indicated times in the absence or presence of FBW7 $\alpha$ overexpression. Total protein was isolated at different times after $\mathrm{CHX}$ treatment, and the levels of c-Myc and cyclin E protein were determined by western blot analysis. The band density of c-Myc and cyclin E protein was quantified by using Scion Imaging software and normalized to $\beta$-actin levels.

MTT assay. CCA cell proliferation was analyzed by MTT assay. A total of $3 \times 10^{3}$ QBC-939 or MZ-chal cells were seeded in 96-well plates and MTT was added to each well every $24 \mathrm{~h}$. The plates were incubated for $4 \mathrm{~h}$ before addition of solubilization solution (10\% SDS in $0.01 \mathrm{M} \mathrm{HCl})$. The absorbance was measured at $560 \mathrm{~nm}$ using a microplate reader.

Focus formation assay. Five hundred cells were cultured in six-well plates in RPMI-1640 with $10 \%$ FBS. Cells were grown for 2-3 weeks, then colonies were stained with $0.05 \%$ crystal violet for $30 \mathrm{~min}$ and counted.

Cell cycle analysis. For cell cycle analysis, $4 \times 10^{5}$ CCA cells were synchronized by serum starvation for $24 \mathrm{~h}$ and induced to re-enter the cell cycle by an exchange of $10 \%$ fetal bovine serum for $9 \mathrm{~h}$. Cells were harvested and fixed in $75 \%$ ethanol at $4^{\circ} \mathrm{C}$ overnight. Cells were incubated with RNase A at $37^{\circ} \mathrm{C}$ for 
$30 \mathrm{~min}$, and then stained with propidium iodide (PI) at $37^{\circ} \mathrm{C}$ for $30 \mathrm{~min}$. Cell cycle was measured by flow cytometry.

Tumor xenograft experiments. After infection by lentivirus, QBC-939 cells were selected with puromycin for 1 week, and then used to inject into nude mice. The protocols for the in vivo studies were approved by Institutional Animal Care and Use Committee of Laboratory Animal Center of Xiamen University. Male nude mice (4-6-week-old) were obtained from Laboratory Animal Center of Xiamen University. Nude mice were injected subcutaneously in both flanks with $4 \times 10^{6}$ QBC-939-FBW7 $\alpha$ and control cells, respectively. Ten days after cell injection, the volume of the tumor was monitored and calculated following the formula: Volume $=$ length $\mathrm{x} \mathrm{Width}^{2} \mathrm{x} 0.52$. After 28 days, tumors were harvested and weighed, and then were dissected and fixed in $10 \%$ formalin and embedded in paraffin for Ki67 staining. Remaining tumors were homogenized in RIPA for western blot analysis.

Immunohistochemistry. Slides were soaked in preheated citrate buffer ( $\mathrm{pH}$ 6.0) and heated in a microwave for $20 \mathrm{~min}$ to retrieve antigen. After cooling, slides were washed with PBS three times, and then incubated with Ki67 antibody (1:200; Invitrogen) overnight at $4^{\circ} \mathrm{C}$. On the second day, slides were washed with PBS three times, and then incubated with a horseradish peroxidase-conjugated secondary antibody for $1 \mathrm{~h}$ at room temperature. After washing, DAB reagent was added to visualize the labeled protein.

Statistical analysis. All data are shown as the mean \pm SD from the number of replicates described in results. The statistically significant effects between mean values $(\mathrm{p}<0.05)$ were assessed with the two-tailed Student's t-test in SPSS.

\section{Results}

Overexpression of $F B W 7 \alpha$ inhibits $C C A$ cell proliferation. To investigate the role of FBW7 $\alpha$ in CCA cell proliferation, the FBW7 $\alpha$-encoding lentivirus was used to express FBW7 $\alpha$ in two CCA cell lines, QBC939 and MZ-Cha1, and then cell proliferation was measured by MTT assay. As shown in Fig. $1 \mathrm{~A}$ and $\mathrm{B}, \mathrm{FBW} 7 \alpha$-encoding lenivirus, but not control lentivirus, efficiently increased the levels of the FBW7 $\alpha$ protein in these cells. Significantly, upregulation of FBW7 $\alpha$ inhibited proliferation of QBC939 and MZ-Cha1 cells (Fig. 1A and B). Furthermore, upregulation of FBW7 $\alpha$ decreased focus formations in QBC939 and MZ-Cha1 cells (Fig. 1C and D). Together, these results indicate that FBW7 $\alpha$ is important for CCA cell proliferation.

We also observed that the decrease in growth of FBW7 $\alpha$-overexpression MZ-Cha1 cells was less than FBW7 $\alpha$ overexpression QBC-939 cells at $96 \mathrm{~h}$ (Fig. 1A and B), the reason may be that the growth of $\mathrm{MZ}$-Cha1 cells is quite nutrition-dependent in culture medium and nutrition reduction in culture medium after $96-\mathrm{h}$ cell culture may result in a reduced growth of control cells. Importantly, our focus forming assay demonstrated that upregulation of FBW7 $\alpha$ significantly decreased focus formations in MZ-Cha1 cells as QBC-939 (Fig. 1C and D), suggesting that FBW7 $\alpha$ has the same tumor suppressor potential in the MZ-Cha1 cells and QBC-939 cells.
To investigate the effect of knockdown of FBW7 $\alpha$ on CCA cell proliferation, RNA interference was used to knock down the expression of FBW7 $\alpha$ in QBC-939 cells and the cell proliferation was measured by MTT assay. Since it is very difficult to detect endogenous FBW7 $\alpha$ protein expression, we use realtime PCR to detect the level of FBW7 $\alpha$ mRNA. As shown in Fig. 1E, FBW7 $\alpha$-specific siRNA, but not control RNA, efficiently reduced the levels of endogenous FBW $7 \alpha$ mRNA. Although downregulation of FBW7 $\alpha$ slightly increased the mean of OD560 in MTT assay at $96 \mathrm{~h}$, they were not statistically significant (Fig. 1F). Since the basal levels of FBW7 $\alpha$ protein were too low in CCA cells, knockdown of FBW7 $\alpha$ could not cause significant changes in cell proliferation.

As upregulation of FBW $7 \alpha$ resulted in a decrease in CCA cell proliferation, cell cycle analysis was performed to detect whether overexpression of FBW7 $\alpha$ induces cell arrest in a specific phase of the cell cycle. The results showed that the percentage of FBW7 $\alpha$-overexpression cells at G1 phase was significantly increased as compared with control cells, and this was associated with a concomitant decrease of cells at the $\mathrm{S}$ and $\mathrm{G} 2 / \mathrm{M}$ of the cell cycle (Fig. 1G and H). These results indicate that upregulation of FBW7 $\alpha$ induces CCA cell arrest in the G1 phase of the cell cycle, which is at least in part responsible for the suppression of cell proliferation by FBW7 $\alpha$.

$F B W 7 \alpha$ promotes the protein degradation of $c-M y c$ and cyclin $E$. To explore the molecular mechanism underlying the crucial role of FBW7 $\alpha$ in control of CCA cell proliferation, we examined the effect of FBW7 $\alpha$ overexpression on the expression of several cell proliferation/survival-related proteins which are identified as the substrates of FBW7 (10-16). As shown in Fig. 2A, overexpression of FBW7 $\alpha$ significantly reduced the protein levels of c-Myc and cyclin E, but had no significant effect on the protein levels of Notch, mTOR, AIB1 and Mcl-1 in either QBC939 or MZ-Cha1 cells. Overexpression of FBW7 $\alpha$ did not affect the mRNA levels of c-Myc and cyclin E (Fig. 2B), indicating that FBW7 $\alpha$ decreases the expression of c-Myc and cyclin E at the post-transcriptional level in CCA cells. It has been reported that c-Myc can inhibit cell cycle inhibitor $\mathrm{p} 21^{\mathrm{CIP} / \mathrm{WAF} 1}$ expression at transcriptional level $(23,24)$. Our results showed that overexpression of FBW7 $\alpha$ increased p $21^{\mathrm{CIP} / \mathrm{WAF} 1}$ expression at both protein and mRNA levels (Fig. 2A and 2B), and downregulation of c-Myc increased p $21^{\mathrm{CIP} / W A F 1}$ expression at both protein and mRNA levels (Fig. 3A) in CCA cells, indicating that FBW7 $\alpha$ increases cell cycle inhibitor $\mathrm{p} 21^{\mathrm{CIP} / \mathrm{WAF} 1}$ expression at least in part through downregulating c-Myc expression.

To determine whether FBW7 $\alpha$ accelerates the degradation of c-Myc and cyclin E proteins, we infected QBC939 and MZ-Cha1 cells with control lentivirus or FBW7 $\alpha$-encoding lentivirus, and then used cycloheximide (CHX) to block protein synthesis. Overexpression of FBW $7 \alpha$ significantly accelerated the degradation of c-Myc and cyclin E proteins when $\mathrm{CHX}$ blocked protein synthesis (Fig. 2C and D), but the effect of FBW $7 \alpha$ on the degradation of c-Myc and cyclin E proteins was suppressed by the proteasome inhibitor MG132 (Fig. 2E). These results indicate that FBW7 $\alpha$ inhibits c-Myc and cyclin E protein expression dependent on ubiquitin-proteasome degradation pathway in CCA cells. This notion is consistent with previous reports in other cells $(10,11)$. 
A
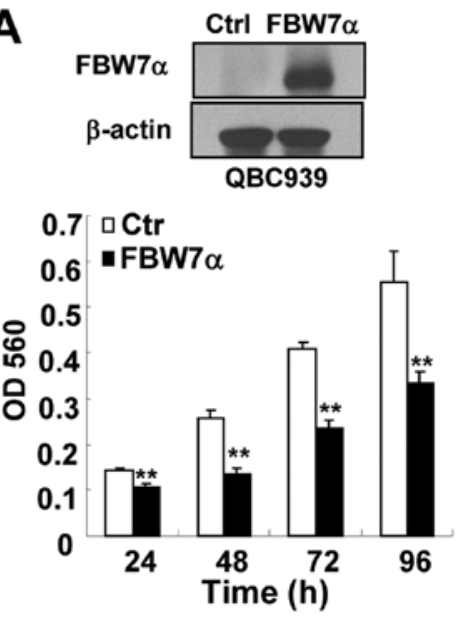

C
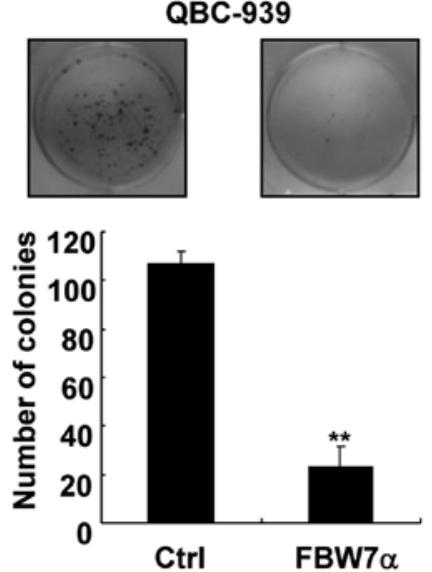

E

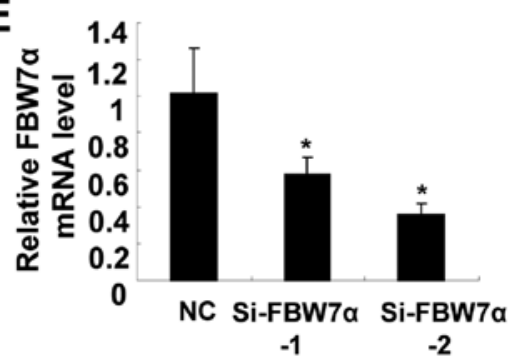

B
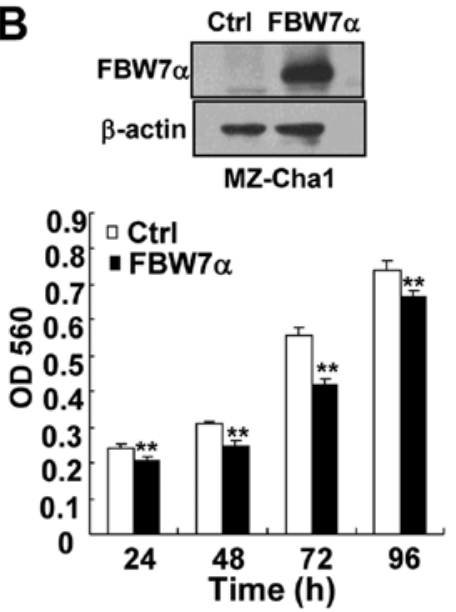

D
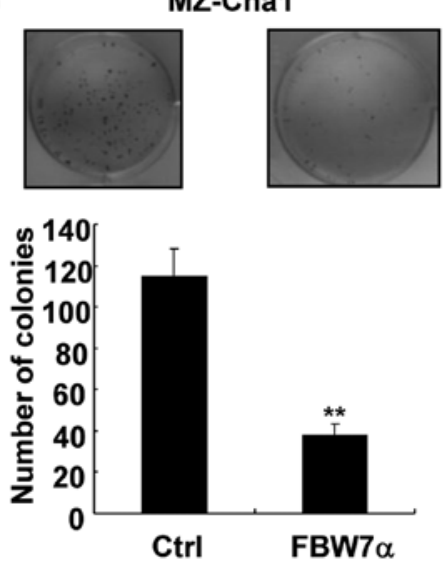

F $0.7 \therefore \mathrm{NC}$
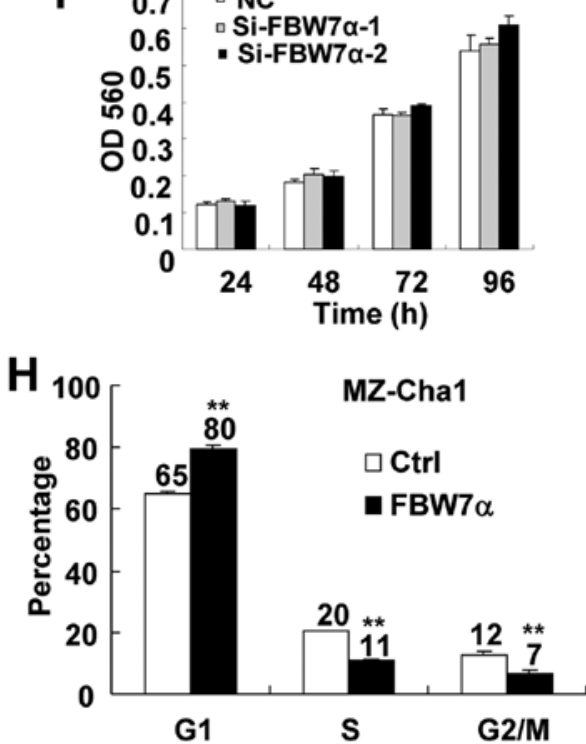

Figure 1. Overexpression of FBW7 $\alpha$ inhibits CCA cell proliferation. CCA cells were infected with control or FBW7 $\alpha$-encoding lentivirus for 48 h and then these cells were used to perform western blot analysis (A and B), MTT assay (A and B), focus formation assay (C and D) and cell cycle analysis (G and H), respectively. QBC-939 cells were transfected with FBW7 $\alpha$-specific siRNA or control RNA for 48 h, then these cells were used to perform Real-time analysis (E) and MTT assay (F). All data are mean $\pm \mathrm{SD}, \mathrm{n}=3,{ }^{*} \mathrm{p}<0.05,{ }^{* *} \mathrm{p}<0.01$.

Downregulation of either -Myc or cyclin E inhibits CCA cell proliferation. To determine whether FBW7 $\alpha$ inhibits CCA proliferation through promoting c-Myc or cyclin $\mathrm{E}$ protein degradation, the effect of downregulation of c-Myc and cyclin $\mathrm{E}$ on CCA proliferation were investigated. We respectively used two different shRNA to knock down c-Myc and cyclin $\mathrm{E}$ in QBC-939 cells, and then measured cell proliferation, focus formation and cell cycle progression. As shown in Fig. 3A, sh-c-Myc and shCyclin E efficiently knocked down the expression of $\mathrm{c}-\mathrm{Myc}$ and cyclin $\mathrm{E}$, and downregulation of $\mathrm{c}-\mathrm{Myc}$ 

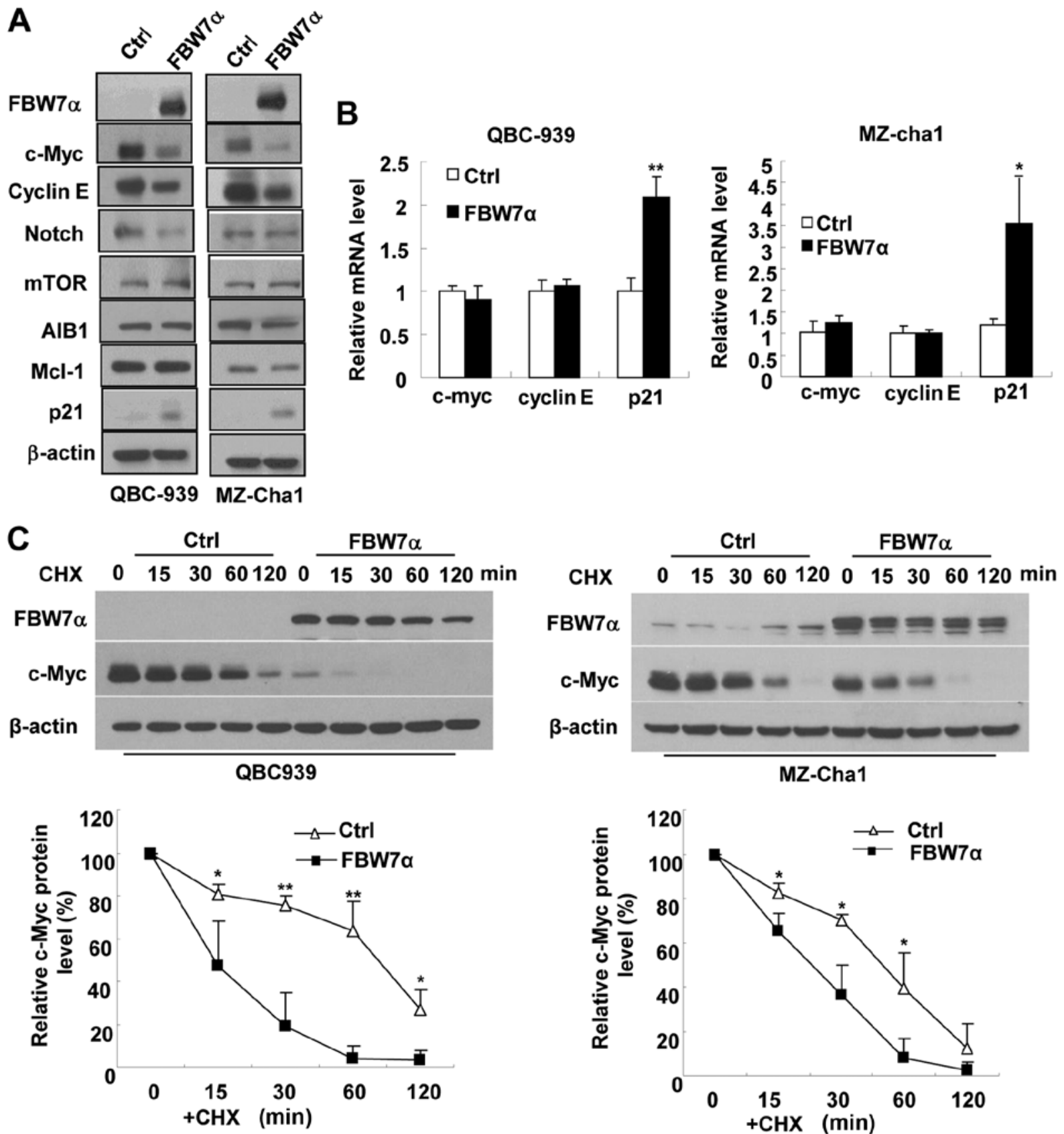

Figure 2. FBW7 $\alpha$ promotes protein degradation of c-Myc and cyclin E. (A and B) CCA cells were infected with control or FBW7 $\alpha$-encoding lentivirus for $72 \mathrm{~h}$, then western blotting and real-time PCR were performed to detect the expression of FBW7 $\alpha$ target genes. (C) Overexpression of FBW7 $\alpha$ promoted protein degradation of $\mathrm{c}-\mathrm{Myc}$ and cyclin $\mathrm{E}$ when cycloheximide $(\mathrm{CHX})$ blocked protein synthesis. All data are mean $\pm \mathrm{SD}, \mathrm{n}=3$.

and cyclin E significantly inhibited CCA cells proliferation, respectively. Downregulation of c-Myc and cyclin E also significantly inhibited focus formation (Fig. 3B), and induced cell cycle arrest in G1 phase (Fig. 3C). These results indicate that downregulation of $\mathrm{c}-\mathrm{Myc}$ and cyclin $\mathrm{E}$ indeed contribute to the inhibitory effect of FBW7 $\alpha$ on CCA proliferation.

Restoration of the expression of $c-M y c$, but not cyclin $E$, partially rescues proliferation of $F B W 7 \alpha$-overexpressing $C C A$ cells. To further determine whether FBW7 $\alpha$ inhibits CCA proliferation through promoting $\mathrm{c}-\mathrm{Myc}$ or cyclin $\mathrm{E}$ protein degradation, FBW7 $\alpha$-overexpressing cells were infected with c-Myc-encoding lentivirus and cyclin E-encoding lentivirus to restore the expression of $\mathrm{c}-\mathrm{Myc}$ and cyclin $\mathrm{E}$, and then cell prolif- eration was measured by MTT assay, respectively. Consistent with the notion of Fig. 2 that FBW7 $\alpha$ could promote c-Myc and cyclin E protein degradation in CCA cells, overexpression of FBW $7 \alpha$ promoted degradation of exogenous c-Myc (compare lanes 2 with lanes 5) or cyclin $\mathrm{E}$ (compare lanes 3 with lanes 6) protein (Fig. 4A). Although, FBW7 $\alpha$ could degrade exogenous c-Myc and cyclin E proteins, as shown in Fig. 4B, infection with c-Myc-encoding lentivirus and cyclin E-encoding lentivirus efficiently restored the expression of c-Myc and cyclin $\mathrm{E}$ in FBW7 $\alpha$-overexpression cells. Restoration of c-Myc expression, but not cyclin E expression, suppressed FBW7 $\alpha$-medicated upregulation of the cell cycle inhibitor $\mathrm{p} 21^{\mathrm{CIP} / \mathrm{WAF} 1}$ in FBW7 $\alpha$ overexpression cells (Fig. 4B). Restoration of c-Myc expression partially rescued the proliferation of FBW $7 \alpha$-overexpression 

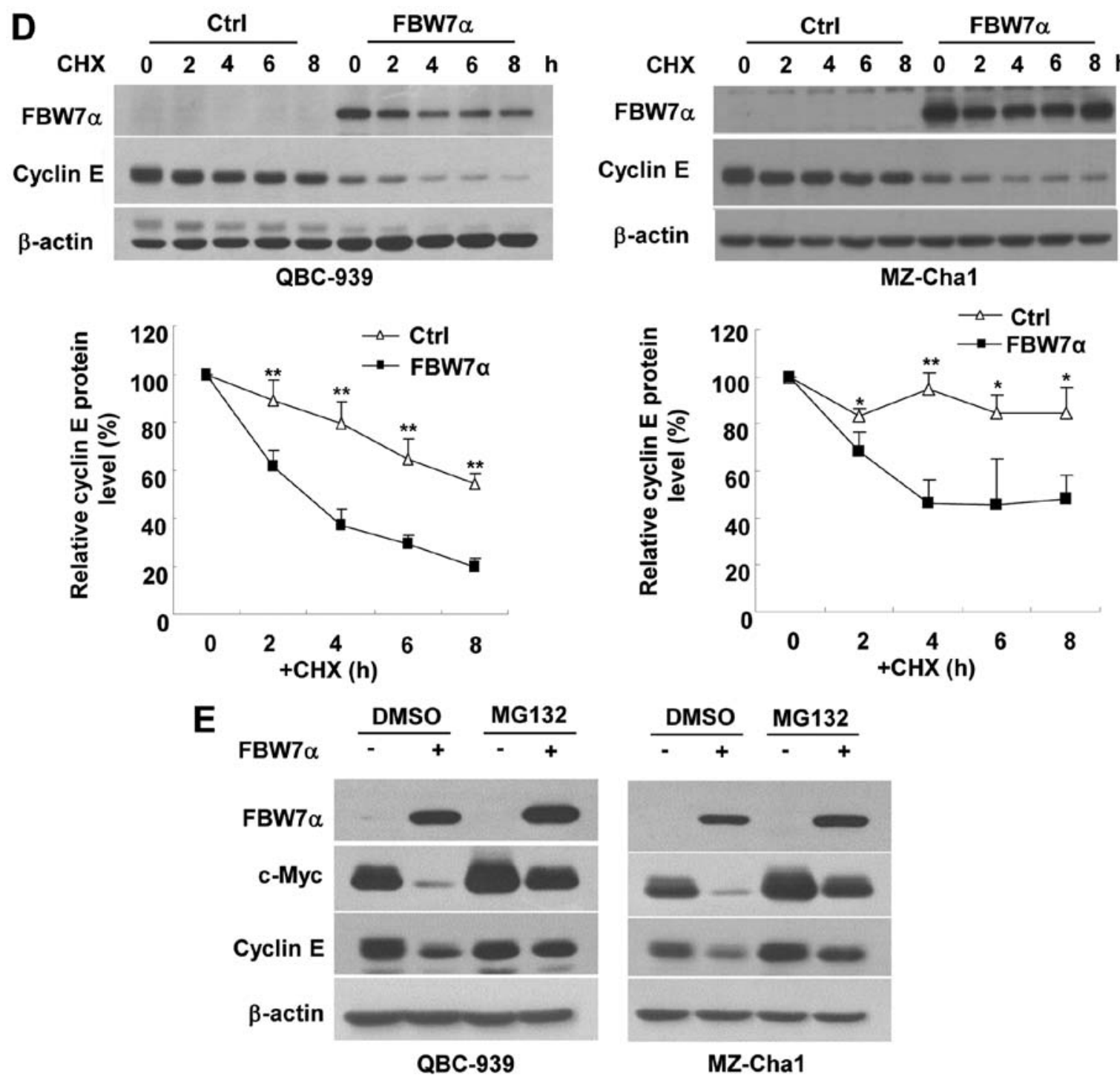

Figure 2. Continued. FBW7 $\alpha$ promotes the protein degradation of c-Myc and cyclin E. (D) Overexpression of FBW7 $\alpha$ promoted the protein degradation of c-Myc and cyclin E when cycloheximide (CHX) blocked protein synthesis. (E) Proteasome inhibitor MG132 inhibited FBW7 $\alpha$-mediated downregulation of the protein levels of c-Myc and cyclin E. All data are mean $\pm S D, n=3$.

cells as expected (Fig. 4C). Surprisingly, restoration of cyclin E expression failed to rescue the proliferation of FBW7 $\alpha$ overexpression cells (Fig. 4C). It may be due to relatively high levels of $\mathrm{p} 21^{\mathrm{CIP} / \mathrm{WAF} 1}$ in FBW7 $\alpha$-overexpression cells (Fig. 4B), which counteracts the promoting effect of cyclin $\mathrm{E}$ on cell proliferation. This notion was supported by the observation that knockdown of $\mathrm{p} 21^{\mathrm{CIP} / \mathrm{WAF} 1}$ partially restored the proliferation of FBW7 $\alpha$-overexpression cells (Fig. 4D and E).

Overexpression of $F B W 7 \alpha$ inhibits CCA tumorigenesis in nude mice. To invistigate the role of FBW7 $\alpha$ in CCA progression in vivo, we examined the effect of FBW7 $\alpha$ overexpression on the growth of CCA xenograft tumors in nude mice injected with FBW7 $\alpha$-overexpression or control QBC939 cells. As shown in Fig. 5A, FBW7 $\alpha$-overexpression CCA tumors grew much slower than control tumors. At the end of study (day 28), tumor weight of FBW7 $\alpha$-overexpression group $(0.0223 \pm 0.0185 \mathrm{~g})$ was only $15 \%$ of the control group $(0.151 \pm 0.0728 \mathrm{~g})$ (Fig. 5B). Western blot results showed that the protein levels of c-Myc and cyclin $\mathrm{E}$ in FBW7 $\alpha$-overexpression CCA tumors were much lower than that of control tumors, whereas $\mathrm{p} 21^{\mathrm{CIP} / \mathrm{WAF} 1}$ expression was much higher (Fig. 5C). Furthermore, immunohistochemical results showed that FBW7 $\alpha$-overexpression CCA tumors had much less Ki67-positive cells ( $\mathrm{Ki}-67$ is known as a marker of cell proliferation) than control tumors (Fig. 5D), indicating that overexpression of FBW7 $\alpha$ inhibits CCA tumor cell proliferation in vivo. Collectively, these results demonstrate that FBW7 $\alpha$ is able to inhibit CCA tumor growth in nude mice.

\section{Discussion}

The mutation rates of FBW7 are relatively tumor typedependent. Cholangiocarcinoma and T-cell acute lymphocytic leukemia harbor 35 and $31 \%$ frequency of mutations for FBW7, respectively, while more than ten other types of tumors (such as stomach, colon, breast, bladder, endometrium) only harbor 0-9\% frequency of mutations (17). Immunohistochemistry staining showed that the expression of FBW7 was downregulated in tumor tissues compared with adjacent non-tumorous 
A
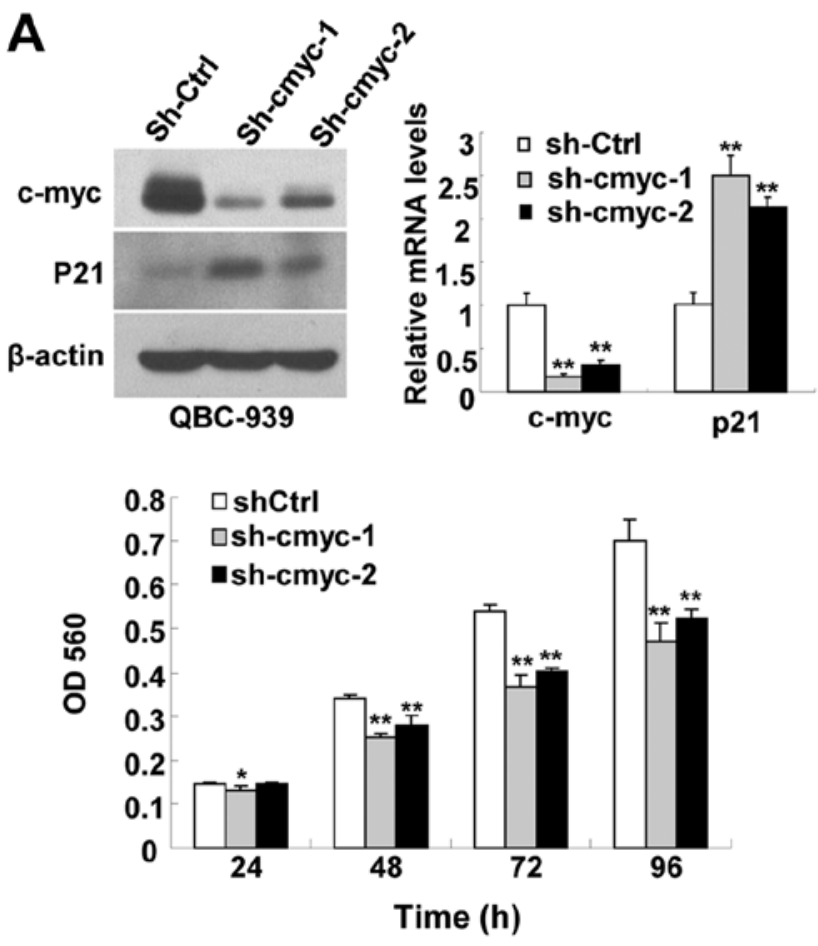

B
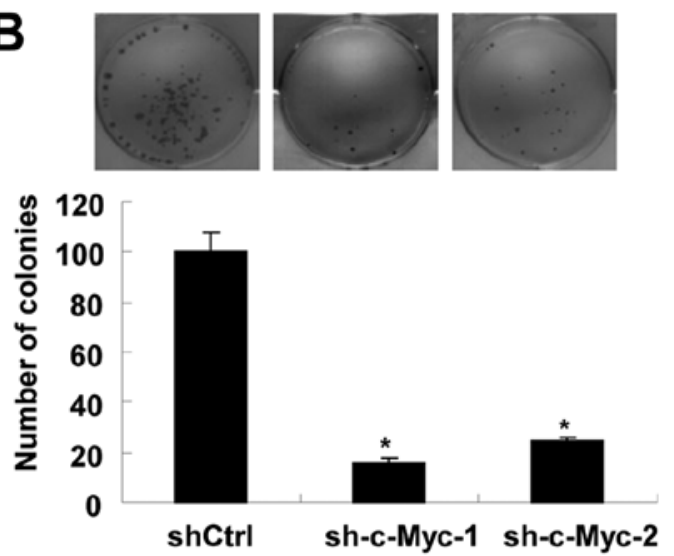

C

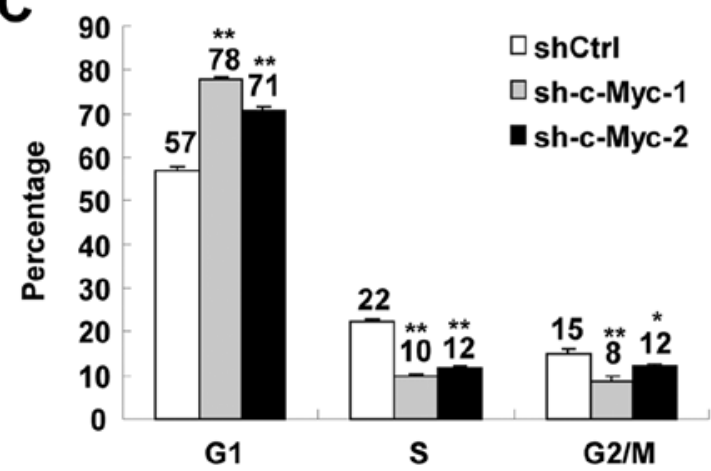

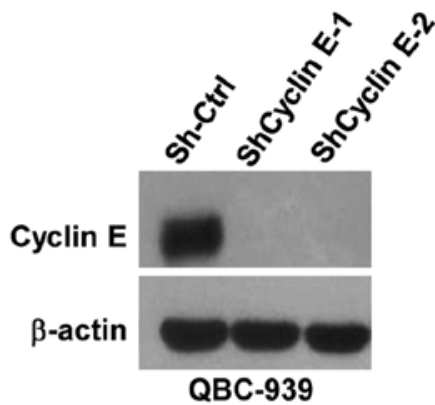
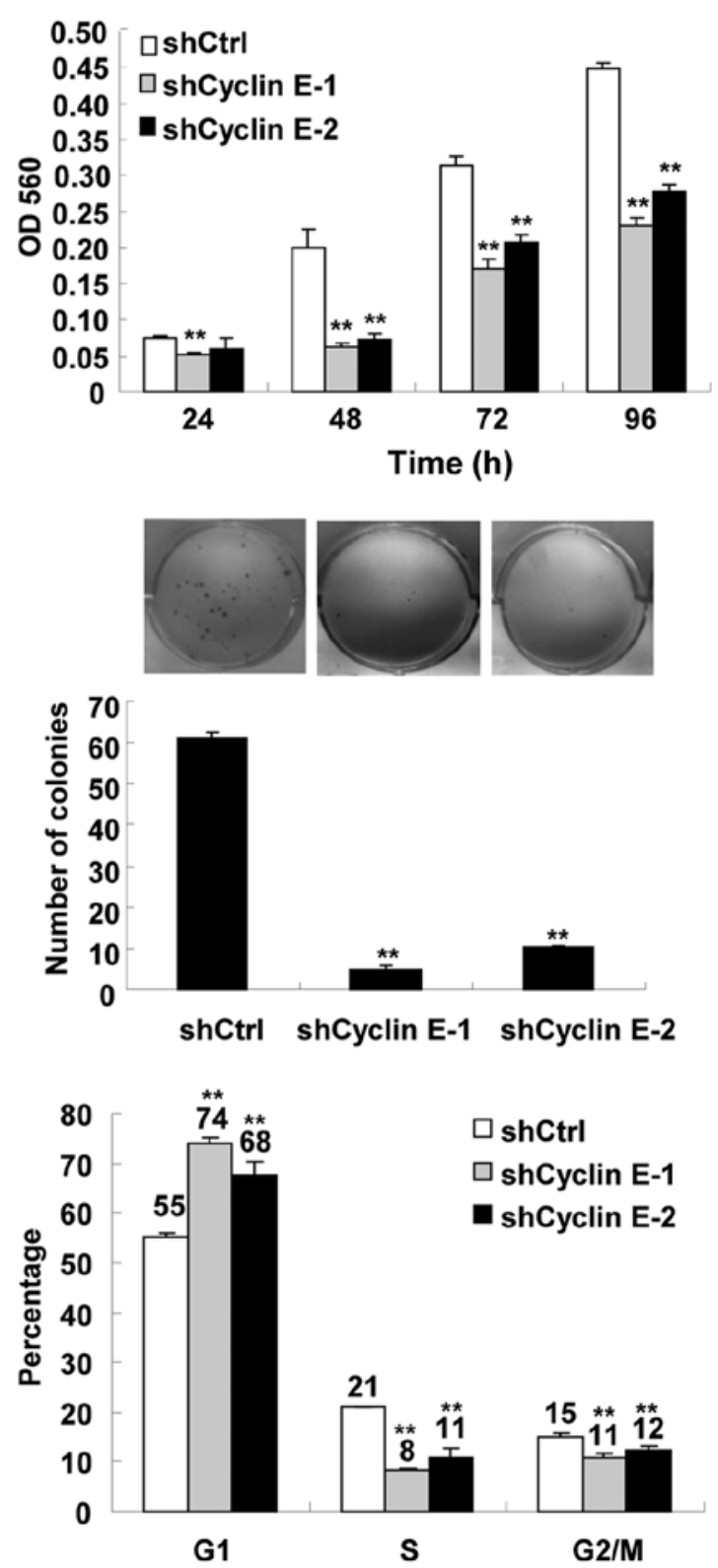

Figure 3. Downregulation of c-Myc or cyclin E inhibits CCA cell proliferation. CCA cells were infected by sh-ctrl, sh-c-Myc-encoding or shCyclin E-encoding lentivirus for $48 \mathrm{~h}$ and these cells were seeded to perform western blotting (A), MTT assay (A), focus formation assay (B) and cell cycle analysis (C), respectively. All data are mean $\pm \mathrm{SD}, \mathrm{n}=3,{ }^{*} \mathrm{p}<0.05,{ }^{* *} \mathrm{p}<0.01$.

tissues in intrahepatic (IHCC) and perihilar (PHCC) CCA (18). The relatively high frequency of impaired FBW7 function in CCA implicate that FBW7 may play a specific role in CCA progression. In the present study, we defined the role of FBW7 in CCA cell proliferation. Our results showed that overexpression of FBW7 $\alpha$ significantly inhibited CCA cell proliferation and CCA xenograft tumor growth, suggesting that FBW7 $\alpha$ plays a tumor suppressor role in CCA progression.

Several oncogenic proteins, such as c-Myc, c-Jun, cyclin E, mTOR, Notch-1, Notch-3, and AIB1, have been identified as the substrates of FBW7 $(4,5)$. However, FBW7 targets different substrates for degradation in a cell type-specific manner and 

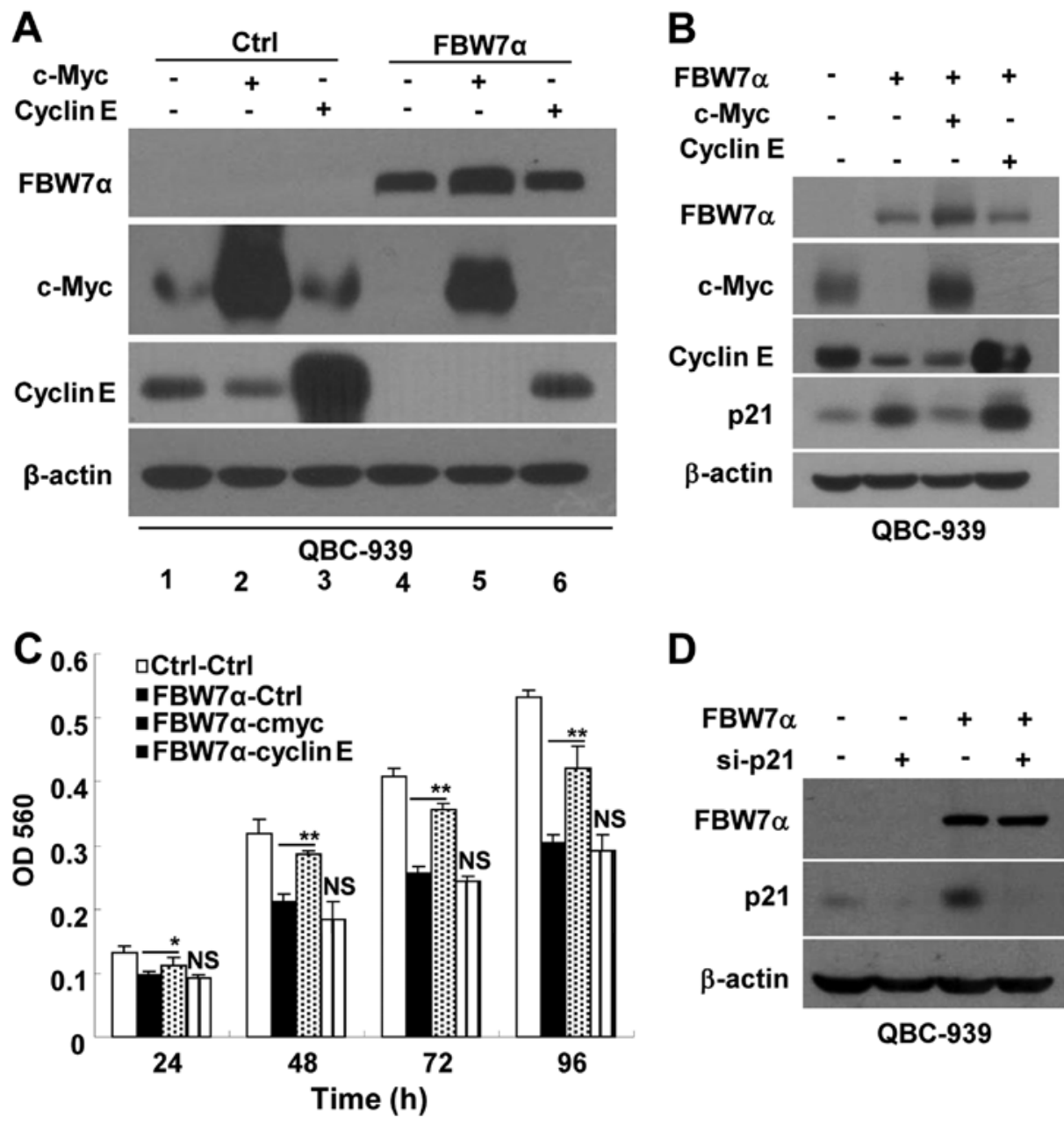

D

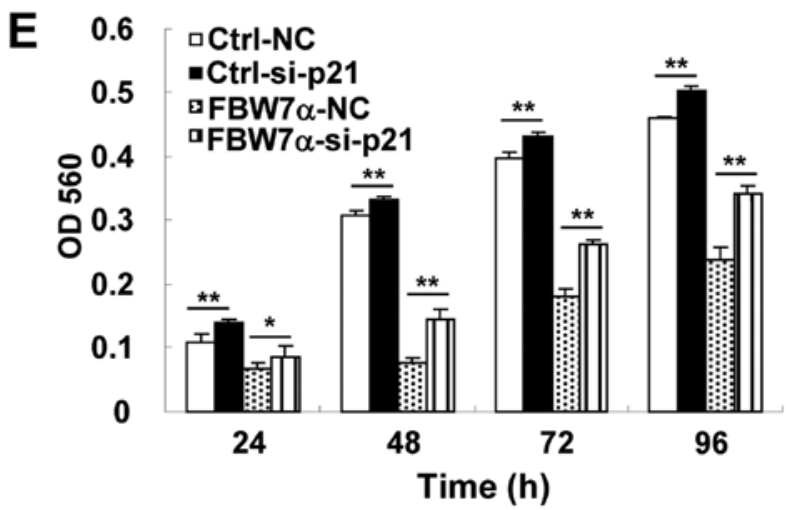

Figure 4. Restoration of the expression of c-Myc, but not cyclin E, partially rescues the proliferation of FBW7 $\alpha$-overexpressing CCA cells. (A) QBC-939 cells were infected with control or FBW7 $\alpha$-encoding lentivirus for $48 \mathrm{~h}$, then control cells and FBW7 $\alpha$-overexpressing cells were infected with control, c-Mycencoding or cyclin E-encoding lentivirus for $24 \mathrm{~h}$, respectively. Then these cells were seeded to perform western blot analysis after $24 \mathrm{~h}$. (B and C) After QBC-939 cells were infected with control or FBW7 $\alpha$-encoding lentivirus for $48 \mathrm{~h}$, the cells were infected with control, c-Myc-encoding or cyclin E-encoding lentivirus for $24 \mathrm{~h}$. Then, QBC-939 cells were seeded to perform western blot analysis (B) and MTT assay (C). (D and E) Knockdown of p21 $1^{\mathrm{CI} / \mathrm{WAF} 1}$ partially restored the proliferation of FBW7 $\alpha$-overexpressing cells. After QBC-939 cells were infected with control or FBW7 $\alpha$-encoding lentivirus for $48 \mathrm{~h}$, these cells were transfected with control siRNA or p21 ${ }^{\mathrm{CI} / \mathrm{WAF} 1}$-specific siRNA for $24 \mathrm{~h}$. Then, QBC-939 cells were seeded to perform western blot analysis (D) and MTT assay (E). Ctrl represents the infection with pLV-CS 2.0-encoding lentivirus; FBW7 $\alpha$ represents the infection with pLV-CS2.0-FBW7 $\alpha$-encoding lentivirus; $\mathrm{NC}$ represents negtive control for si-p21; si-p21 represents p21-specific siRNA. All data are mean $\pm \mathrm{SD}, \mathrm{n}=3,{ }^{*} \mathrm{p}<0.05,{ }^{* *} \mathrm{p}<0.01$.

the role of FBW7 in cell proliferation may depend on cell and signaling context. For example, conditional deletion of FBW7 in mouse keratinocytes significantly increased the protein levels of Notch1 and c-Myc, but not c-Jun, Notch3 and cyclin E (25). FBW7 deletion in mouse thymocytes significantly increased the expression of Notch1, Notch3 and
c-Myc, but not cyclin E (26). Although conditional deletion of FBW7 in hematopoietic stem cells, keratinocytes or T cells promoted cell proliferation (25-27), FBW7 deletion inhibited cell proliferation in mouse embryonic fibroblasts $(28,29)$. In the present study, we found that the decrease in the amounts of c-Myc and cyclin E (rather than mTOR, Notch, AIB1 and 

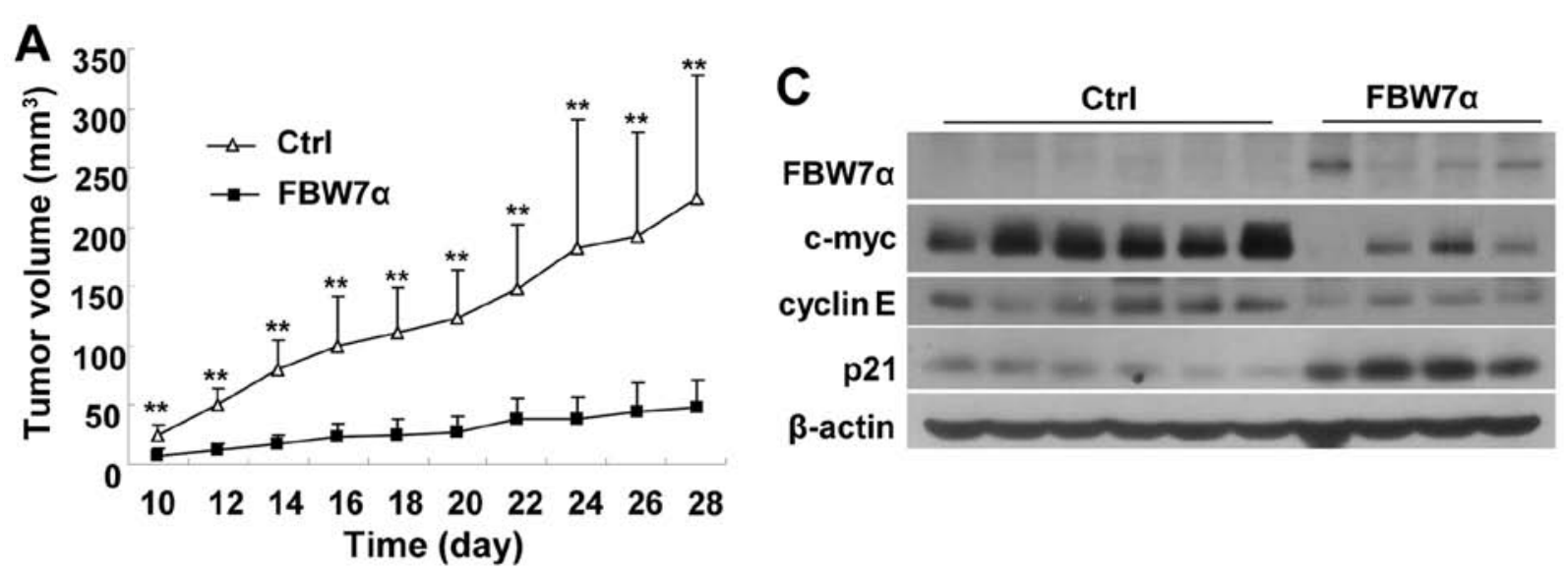

B

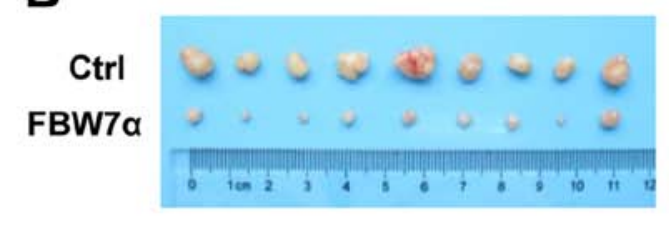

D
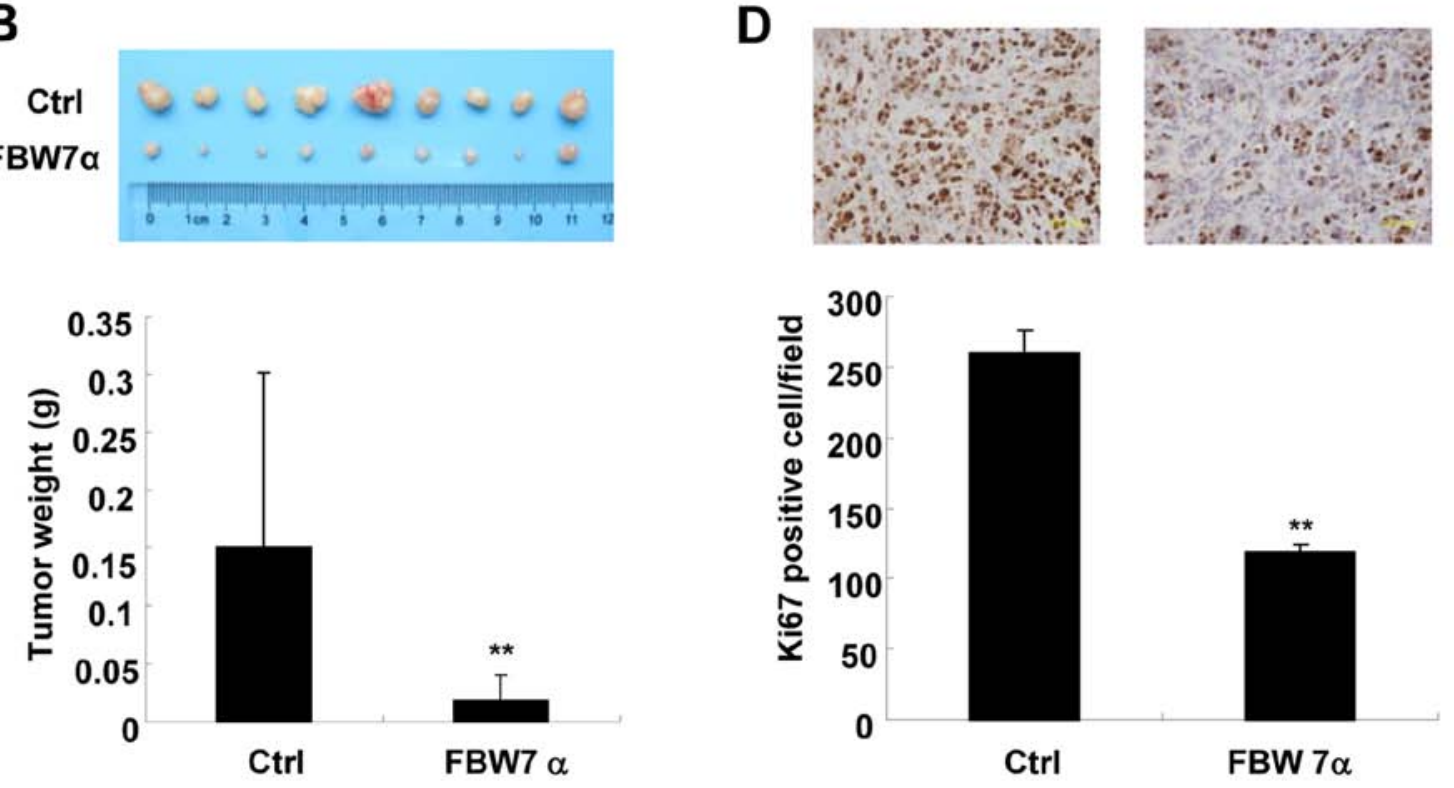

Figure 5. Overexpression of FBW7 $\alpha$ inhibits CCA tumorigenesis in nude mice. (A) Overexpression of FBW7 $\alpha$ inhibited CCA tumor growth. $\mathrm{n}=9$; ${ }^{* *} \mathrm{p}<0.01$. (B) Tumor weight was quantitated at the end of the experiment. $n=9 ;{ }^{* *} \mathrm{p}<0.01$. (C) Related protein levels in tumors were detected by western blot analysis at the end of the experiment. (D) Overexpression of FBW7 $\alpha$ inhibited tumor cell proliferation. Tumor cell proliferation was detected by Ki- $67 \mathrm{staining}$. $\mathrm{n}=4$; ${ }^{* *} \mathrm{p}<0.01$.

Mcl-1) in FBW7 $\alpha$-overexpression CCA cells were the most pronounced and reproducible in different CCA cell lines, suggesting that downregulation of c-Myc and cyclin E may mediate the inhibitory effect of FBW7 $\alpha$ in CCA cell proliferation. This notion is supported by the observation that knockdown of c-Myc or cyclin E significantly inhibited CCA cell proliferation. Noteworthy, while restoration of c-Myc expression in FBW7 $\alpha$-overexpressing CCA cells partially rescued the cell proliferation, restoration of cyclin E expression in FBW7 $\alpha$-overexpressing CCA cells failed to rescue the cell proliferation. Restoration of c-Myc expression in FBW7 $\alpha$-overexpression CCA cells could significantly downregulate $\mathrm{p} 21^{\mathrm{CIP} / \mathrm{WAF} 1}$ expression, but restoration of cyclin $\mathrm{E}$ expression in FBW7 $\alpha$-overexpression CCA cells could not downregulate $\mathrm{p} 21^{\mathrm{CIP} / \mathrm{WAF} 1}$ expression. These results suggest that high level of $\mathrm{p} 21^{\mathrm{CIP} / \mathrm{WAF} 1}$ could abolish the promotive effect of cyclin $\mathrm{E}$ on proliferation in cyclin E/FBW7 $\alpha$ overexpression CCA cells. Downregulation of $\mathrm{p} 21^{\mathrm{CIP} / \mathrm{WAF} 1}$ in FBW7 $\alpha$-overexpressing CCA cells could partially rescue the cell proliferation.

Our study demonstrated that FBW7 $\alpha$ plays an essential inhibitory role in CCA progression, suggesting that targeting FBW7 $\alpha$ pathway becomes an attracting strategy in CCA treatment. Our results showed that restoration of the expression of c-Myc partially rescues proliferation of FBW7 $\alpha-$ overexpressing CCA cells, suggesting that downregulation of c-Myc is partially responsible for FBW7 $\alpha$-induced inhibition of CCA cell proliferation. Consistent with our results, cremediated ablation of c-Myc (but not inactivation of notch signaling) prevented the stimulatory effect of FBW7 ablation on keratinocyte or $\mathrm{T}$ cell proliferation in mice $(25,26)$, suggesting that FBW7-c-Myc axis is important for controlling cell proliferation. The expression of FBW7 $\alpha$ substrate c-Myc was induced in different animal models of CCA and promoted CCA progression (30-32), suggesting that c-Myc plays an important role in CCA progression.

It has been reported that FBW7 hotspot mutants in human CCA not only reveal defects in localization and substrate binding, but also can abrogate wild-type FBW7 function through a dominant negative mechanism (17). Since mutated endogenous FBW7 proteins are not only functionally inactivated, but also impair wild-type FBW7 function, it is not a good strategy to target FBW7 directly for CCA treatment. Given that our results demonstrate that downregulation of c-Myc expres- 
sion plays an important role in inhibition of proliferation of FBW7 $\alpha$-overexpression CCA cells, therefore, targeting FBW7 $\alpha$ substrate c-Myc with a pharmacological c-Myc inhibitor may be a viable strategy for CCA treatment. It has been reported that a small molecular c-Myc inhibitor 10058-F4 could induce p $21^{\mathrm{CIP} / \mathrm{WAF} 1}$ expression, inhibit tumor cell proliferation and enhance chemosensitivity of human hepatocellular carcinoma cells and acute myeloid leukemia $(33,34)$. Therefore, c-Myc inhibitor may represent a promising therapeutic approach for CCA with FBW7 mutated or downregulated.

\section{Acknowledgements}

This work was supported by grants from the Natural Science Foundation of China (no. 81272246 to W.L.), the Xiamen Science and Technology Plan (no. $3502 Z 20154037$ to M.L.) and Natural Science Foundation of Fujian Province (no. 2015J01502 to Z.Z.).

\section{References}

1. Seeree P, Pearngam P, Kumkate $S$ and Janvilisri T: An omics perspective on molecular biomarkers for diagnosis, prognosis, and therapeutics of cholangiocarcinoma. Int J Genomics 2015: $179528,2015$.

2. Razumilava N and Gores GJ: Cholangiocarcinoma. Lancet 383: 2168-2179, 2014

3. Bergquist A and von Seth E: Epidemiology of cholangiocarcinoma. Best Pract Res Clin Gastroenterol 29: 221-232, 2015.

4. Welcker M and Clurman BE: FBW7 ubiquitin ligase: A tumour suppressor at the crossroads of cell division, growth and differentiation. Nat Rev Cancer 8: 83-93, 2008.

5. Wang L, Ye X, Liu Y, Wei W and Wang Z: Aberrant regulation of FBW7 in cancer. Oncotarget 5: 2000-2015, 2014

6. Spruck CH, Strohmaier H, Sangfelt O, Müller HM, Hubalek M, Müller-Holzner E, Marth C, Widschwendter M and Reed SI: hCDC4 gene mutations in endometrial cancer. Cancer Res 62: 4535-4539, 2002.

7. Welcker M, Orian A, Grim JE, Eisenman RN and Clurman BE A nucleolar isoform of the Fbw7 ubiquitin ligase regulates c-Myc and cell size. Curr Biol 14: 1852-1857, 2004.

8. Grim JE, Gustafson MP, Hirata RK, Hagar AC, Swanger J, Welcker M, Hwang HC, Ericsson J, Russell DW and Clurman BE: Isoform- and cell cycle-dependent substrate degradation by the Fbw7 ubiquitin ligase. J Cell Biol 181: 913-920, 2008.

9. Matsumoto A, Onoyama I and Nakayama KI: Expression of mouse Fbxw7 isoforms is regulated in a cell cycle- or p53-dependent manner. Biochem Biophys Res Commun 350: 114-119, 2006.

10. Yada M, Hatakeyama S, Kamura T, Nishiyama M, Tsunematsu R, Imaki H, Ishida N, Okumura F, Nakayama K and Nakayama KI: Phosphorylation-dependent degradation of c-Myc is mediated by the F-box protein Fbw7. EMBO J 23: 2116-2125, 2004.

11. Koepp DM, Schaefer LK, Ye X, Keyomarsi K, Chu C, Harper JW and Elledge SJ: Phosphorylation-dependent ubiquitination of cyclin $\mathrm{E}$ by the SCFFbw7 ubiquitin ligase. Science 294: 173-177, 2001.

12. Mao JH, Kim IJ, Wu D, Climent J, Kang HC, DelRosario R and Balmain A: FBXW7 targets mTOR for degradation and cooperates with PTEN in tumor suppression. Science 321: 1499-1502, 2008.

13. Inuzuka H, Shaik S, Onoyama I, Gao D, Tseng A, Maser RS, Zhai B, Wan L, Gutierrez A, Lau AW, et al: SCF(FBW7) regulates cellular apoptosis by targeting MCL1 for ubiquitylation and destruction. Nature 471: 104-109, 2011.

14. Tsunematsu R, Nakayama K, Oike Y, Nishiyama M, Ishida N, Hatakeyama S, Bessho Y, Kageyama R, Suda T and Nakayama KI: Mouse Fbw7/Sel-10/Cdc4 is required for notch degradation during vascular development. J Biol Chem 279: 9417-9423, 2004.
15. Wu G, Lyapina S, Das I, Li J, Gurney M, Pauley A, Chui I, Deshaies RJ and Kitajewski J: SEL-10 is an inhibitor of notch signaling that targets notch for ubiquitin-mediated protein degradation. Mol Cell Biol 21: 7403-7415, 2001.

16. Wu RC, Feng Q, Lonard DM and O'Malley BW: SRC-3 coactivator functional lifetime is regulated by a phospho-dependent ubiquitin time clock. Cell 129: 1125-1140, 2007.

17. Akhoondi S, Sun D, von der Lehr N, Apostolidou S, Klotz K, Maljukova A, Cepeda D, Fiegl H, Dafou D, Marth C, et al: FBXW7/hCDC4 is a general tumor suppressor in human cancer. Cancer Res 67: 9006-9012, 2007.

18. Yang H, Lu X, Liu Z, Chen L, Xu Y, Wang Y, Wei G and Chen Y: FBXW7 suppresses epithelial-mesenchymal transition, stemness and metastatic potential of cholangiocarcinoma cells. Oncotarget 6: 6310-6325, 2015.

19. Barsotti AM and Prives C: Pro-proliferative FoxM1 is a target of p53-mediated repression. Oncogene 28: 4295-4305, 2009.

20. Yang C, Li S, Wang M, Chang AK, Liu Y, Zhao F, Xiao L, Han L, Wang D, Li S, et al: PTEN suppresses the oncogenic function of AIB1 through decreasing its protein stability via mechanism involving Fbw7 alpha. Mol Cancer 12: 21, 2013.

21. van Drogen F, Sangfelt O, Malyukova A, Matskova L, Yeh E, Means AR and Reed SI: Ubiquitylation of cyclin $E$ requires the sequential function of SCF complexes containing distinct hCdc4 isoforms. Mol Cell 23: 37-48, 2006.

22. Wang R, Wang Y, Liu N, Ren C, Jiang C, Zhang K, Yu S, Chen Y, Tang H, Deng Q, et al: FBW7 regulates endothelial functions by targeting KLF2 for ubiquitination and degradation. Cell Res 23: 803-819, 2013

23. Gartel AL and Radhakrishnan SK: Lost in transcription: p21 repression, mechanisms, and consequences. Cancer Res 65: 3980-3985, 2005.

24. Bretones G, Delgado MD and León J: Myc and cell cycle control. Biochim Biophys Acta 1849: 506-516, 2015.

25. Ishikawa Y, Hosogane M, Okuyama R, Aoyama S, Onoyama I Nakayama KI and Nakayama K: Opposing functions of Fbxw7 in keratinocyte growth, differentiation and skin tumorigenesis mediated through negative regulation of $\mathrm{c}-\mathrm{Myc}$ and Notch. Oncogene 32: 1921-1932, 2013.

26. Onoyama I, Tsunematsu R, Matsumoto A, Kimura $T$, de Alborán IM, Nakayama K and Nakayama KI: Conditional inactivation of Fbxw7 impairs cell-cycle exit during T cell differentiation and results in lymphomatogenesis. J Exp Med 204: 2875-2888, 2007.

27. Matsuoka S, Oike Y, Onoyama I, Iwama A, Arai F, Takubo K, Mashimo Y, Oguro H, Nitta E, Ito K, et al: Fbxw7 acts as a critical fail-safe against premature loss of hematopoietic stem cells and development of T-ALL. Genes Dev 22: 986-991, 2008.

28. Ishikawa Y, Onoyama I, Nakayama KI and Nakayama K: Notchdependent cell cycle arrest and apoptosis in mouse embryonic fibroblasts lacking Fbxw7. Oncogene 27: 6164-6174, 2008.

29. Masuda K, Ishikawa Y, Onoyama I, Unno M, de Alborán IM, Nakayama KI and Nakayama K: Complex regulation of cell-cycle inhibitors by Fbxw7 in mouse embryonic fibroblasts. Oncogene 29: 1798-1809, 2010

30. Yang H, Li TW, Ko KS, Xia M and Lu SC: Switch from Mnt-Max to Myc-Max induces p53 and cyclin D1 expression and apoptosis during cholestasis in mouse and human hepatocytes. Hepatology 49: 860-870, 2009

31. Prakobwong S, Khoontawad J, Yongvanit P, Pairojkul C, Hiraku Y, Sithithaworn P, Pinlaor P, Aggarwal BB and Pinlaor S: Curcumin decreases cholangiocarcinogenesis in hamsters by suppressing inflammation-mediated molecular events related to multistep carcinogenesis. Int J Cancer 129: 88-100, 2011.

32. Yang H, Li TW, Peng J, Tang X, Ko KS, Xia M and Aller MA: A mouse model of cholestasis-associated cholangiocarcinoma and transcription factors involved in progression. Gastroenterology 141: 378-388, 388.e1-4, 2011.

33. Lin CP, Liu JD, Chow JM, Liu CR and Liu HE: Small-molecule c-Myc inhibitor, 10058-F4, inhibits proliferation, downregulates human telomerase reverse transcriptase and enhances chemosensitivity in human hepatocellular carcinoma cells. Anticancer Drugs 18: 161-170, 2007.

34. Huang MJ, Cheng YC, Liu CR, Lin S and Liu HE: A smallmolecule c-Myc inhibitor, 10058-F4, induces cell-cycle arrest, apoptosis, and myeloid differentiation of human acute myeloid leukemia. Exp Hematol 34: 1480-1489, 2006. 